\title{
ACUTE AND SUB-ACUTE TOXICITY EVALUATION OF ENTEROCOCCUS FAECALIS HZNU P2 ISOLATED FROM PEACOCK FAECES IN VIVO
}

\author{
Y. ZHANG ${ }^{a}$, W. ZHENG ${ }^{a}$, Q.C. YING ${ }^{a}$, L.-E. SHI ${ }^{a}$, Z.-L. ZHANG ${ }^{a}$, \\ M.-Z. SHI ${ }^{b}$ and Z.-X. TANG ${ }^{\text {* }}$ \\ ${ }^{a}$ College of Life and Environmental Sciences, Hangzhou Normal University, 310016, 16\#, Xuelin Street, \\ Hangzhou, Zhejiang. China \\ ${ }^{\mathrm{b}}$ Hangzhou Tianlong Group Co. Ltd, 310021, 1188\#, Linding Street, Hangzhou, Zhejiang. China \\ (Received: 17 December 2015; accepted: 24 February 2016)
}

\begin{abstract}
The sub-acute toxicity of E. faecalis HZNU P2 was investigated in rats fed with different doses for 14 days. To evaluate the acute oral toxicity of $E$. faecalis HZNU P2, rats were fed with E. faecalis HZNU P2 at a high dose of $2 \times 10^{11} \mathrm{CFU} \mathrm{kg}{ }^{-1}$ for 10 days. Results showed that there were no abnormal clinical signs in any of the groups during the experiment. There were no significant differences in live weight gain among rats fed with E. faecalis HZNU P2, compared to those in control group. Macroscopic or microscopic examinations of organs revealed no abnormalities, indicating that $E$. faecalis HZNU P2 did not adversely affect the health of rats. Results of this study demonstrated that digestion of $E$. faecalis HZNU P2 in rats did not show any obvious signs of toxicity.
\end{abstract}

Keywords: Enterococcus faecalis, safety, toxicity, probiotics, rats

Probiotics are live microorganisms that can confer health benefit on the host when administered in adequate amounts (YAKABE et al., 2009, 2011; SHI et al., 2013). Many studies have shown that probiotics can prevent diarrhoea and many other diseases (HAMILTON-MilLER, 2004; BAI \& Ouyang, 2006; Mach, 2006; Ouwehand, 2007; McFarland \& Dublin, 2008; Yakabe et al., 2009, 2011), balance intestinal microflora, and stimulate the immune system (Кнан, 2007). Recently, probiotics have received increasing attention, and many health-promoting probiotic-based products have been developed. As a result, more and more new specific strains of probiotic bacteria are being screened and identified.

Lactic acid bacteria (LAB) have been ingested safely for many centuries and now have been widely used in food relevant fields (ZhOu et al., 2000; YAKABE et al., 2009, 2011). Many LAB strains, such as Lactobacillus, Bifidobacterium, Streptococcus, and Enterococcus spp., have abilities to inhibit the growth of many intestinal pathogens in human and animals (TsAI et al., 2004). This anti-microbiological action is related to many factors, including the production of $\mathrm{H}_{2} \mathrm{O}_{2}$, organic acids, and bacteriocins (NAKAI \& SIEBERT, 2003; Lin et al., 2007; KIRTZALIDOU et al., 2011). Of LAB strains used as probiotics, there is some debate about the genus Enterococcus (TsaI et al., 2004). It has been isolated from many sources such as milk and cheese samples, human vaginal secretions, the preen gland secretion of birds, and animal faeces (Medina et al., 2001; LinaJe et al., 2004; Tsai et al., 2004; Laukova et al., 2008; MARTIN et al., 2008; Soler et al., 2008). It can prevent various gastrointestinal infections and acute enteritis (TsaI et al., 2004). However, a potential risk associated with Enterococcus

\footnotetext{
* To whom correspondence should be addressed. Phone: +86-571-86015696, e-mail: tangzhenxing@126.com
} 
strains has been reported (SAFDAR et al., 2002; Gomes et al., 2008). Thus, before any Enterococcus strains can be incorporated into products for human or animal consumption, the safety of it should be carefully assessed and tested.

Recently, a new strain, E. faecalis HZNU P2, from peacock faeces has been isolated by our group, which has good tolerance to $\mathrm{NaCl}$, low $\mathrm{pH}$, and to simulated gastro-intestinal tract. In addition, E. faecalis HZNU P2 adhere to Hep-2 cells well, and exhibit antimicrobial activity against selected pathogens (results not published). It has a good potential for use as a probiotic in food or feed industries. In this study, to further assess the safety of this potential probiotic, the study of acute and sub-acute toxicity of E. faecalis HZNU P2 was performed in the rats. The effect of consumption of E. faecalis HZNU P2 on the health status of rats was studied. All the results obtained in this work will build a basis for the application of $E$. faecalis HZNU P2.

\section{Materials and methods}

\subsection{Bacterial strains}

Fresh peacock faecal samples (Hangzhou Normal University, Zhejiang, China) were collected, transported to the lab within $2 \mathrm{~h}$, and kept refrigerated $\left(4^{\circ} \mathrm{C}\right)$ until the analysis. Faecal samples were diluted with sterile saline water $(0.85 \%)$. A $100 \mu \mathrm{l}$ diluted faecal samples were transferred to $10 \mathrm{ml}$ bile salt broth at $37^{\circ} \mathrm{C}$ for $24 \mathrm{~h}$. Afterwards, serial 10 -fold dilution of the culture was plated onto de Man, Rogosa and Sharpe (MRS, Oxoid) agar supplemented with $2.0 \% \mathrm{CaCO}_{3}$. The plates were incubated at $37^{\circ} \mathrm{C}$ for $24 \mathrm{~h}$. The formed colonies on the plates were randomly picked and streaked on fresh MRS agar plates by dilution streaking to obtain single colonies. Pure culture was stored at $-80^{\circ} \mathrm{C}$ in MRS broth supplemented with $50 \%$ glycerol. The strain E. faecalis HZNU P2 was identified through biochemical studies, and $16 \mathrm{~S}$ identification according to our previous work (ZHENG et al., 2015) was used in this study. It was deposited in China Center for Type Culture Collection (CСTCC) under the preservation number CCTCC M 2014197. Stock strain was propagated in MRS broth for 24 $\mathrm{h}$ and then concentrated by centrifugation. The cell pellets were re-suspended in saline solution at three concentrations $\left(2 \times 10^{11} \mathrm{CFU} \mathrm{ml}^{-1}, 5 \times 10^{10} \mathrm{CFU} \mathrm{ml}^{-1}, 1 \times 10^{10} \mathrm{CFU} \mathrm{m}^{-1}\right)$ after washing three times by sterile water. Fresh saline water was used for each sample.

\subsection{Animals}

Sprague-Dawley rats $(25 \pm 5 \mathrm{~g})$ were bred at the Animal Unit of Hangzhou Normal University. The rats were examined for health status on arrival and observed for 3 days before the experiments. After 3 days of acclimation, the rats were housed under relative humidity of $45 \sim 50 \%$ and room temperature between 20 and $25{ }^{\circ} \mathrm{C}$, with $12 \mathrm{~h}$ light-dark cycle. All procedures were in accordance with the Guidelines of the Animal Care and Use of Laboratory Animals from the Association of Laboratory Animal Science and the Center for Laboratory Animal Science of Hangzhou Normal University.

\subsection{Acute toxicity}

For the acute toxicity assay, 40 healthy males and females were selected and randomly divided into 2 groups (10 rats of each sex in each group). The test strain was suspended in saline solution to make a concentration of $2 \times 10^{11} \mathrm{CFU} \mathrm{kg}^{-1}$. An oral dose of $2 \times 10^{11} \mathrm{CFU} \mathrm{kg}^{-1}$ 
test strain was administrated to the treatment group and saline solution was administrated to the control group twice by oral gavage $(0 / 12 \mathrm{~h})$ within $24 \mathrm{~h}$. Following administration, clinical signs of the rats were observed for 10 days. At the end of the test, all rats were fasted for $12 \mathrm{~h}$ before anesthetization. The necropsy for the rats was performed.

\subsection{Sub-acute toxicity}

For the sub-acute toxicity assay, 40 healthy males and females were selected and randomly divided into 4 groups ( 5 rats of each sex in each group). The test strain was suspended in saline solution to make at a concentration of $2 \times 10^{11} \mathrm{CFU} \mathrm{kg}{ }^{-1}$ (as high dose), $5 \times 10^{10} \mathrm{CFU} \mathrm{kg}^{-1}$ (as medium dose), and $1 \times 10^{10} \mathrm{CFU} \mathrm{kg}^{-1}$ (as low dose). The test strain and saline solution was administrated to the treatment group and the control group by oral gavage, once per day for 14 days, respectively. Following administration, clinical signs of the rats were observed every day. At the end of the test, all the rats were fasted for $12 \mathrm{~h}$ before anaesthetization. The necropsy for the rats was performed.

\subsection{Clinical observations}

Health status, such as abnormalities, activity, hair lustre, and mortality were observed every day.

\subsection{Body weight}

The body weight of each rat was measured every day.

\subsection{Haematological and plasma biochemical examination}

The animals were fasted overnight before blood collection. Blood samples were drawn into separate tubes containing ethylenediaminetetraacetic acid (EDTA). A PC-603 Haematology Analyser was used to determine haemoglobin concentration (HB), red blood cells (RBC), white blood cells (WBC), lymphocytes (Ly), neutrophils (Ne), and monocytes (Mo). Blood samples were centrifuged to obtain plasma. The biochemical parameters of plasma, including total protein (TP), glucose (GLU), cholesterol (CHO), and triglyceride (TG), were analysed by a biochemical analyser (TBA-FR40, Toshiba Co. Ltd, Osaka, Japan).

\subsection{Necropsy}

Following the sacrifice, a thorough necropsy was performed on all rats. The organs (renal, liver, spleen) were weighed. The organ to body weight ratios (relative organ weights) were calculated from the absolute organ weights and the terminal body weight of the rats. Samples of the weighted organs were washed by saline water and preserved in $10 \%$ formaldehyde solution. Histopathologic analysis was conducted on paraffin-embedded tissues stained with haematoxylin and eosin. The preserved organs were observed by microscopy.

\subsection{Statistics}

Statistical analysis was performed using Origin 8.0 for Windows. The results are presented in mean value \pm standard deviation (SD). The significance of difference was evaluated using one-way analysis of variance. Duncan's test was used to compare the significance of differences in this paper. The differences were considered significant at the level of $\mathrm{P}<0.05$. 


\section{Results and discussion}

As stated before, many strains of probiotic bacteria have been commercialized in food or feed industries. However, the safety of any newly isolated strains should be studied before their incorporation into the products. The focus of this paper is to evaluate the safety of the strain E. faecalis HZNU P2 isolated from peacock faeces. A 10-days acute toxicity and a 14-days sub-acute toxicity of $E$. faecalis HZNU P2 were evaluated in rats.

\subsection{Acute toxicity}

Acute oral toxicity, used as a fundamental test for the safety evaluation, has been reported previously by many researchers (HuANG et al., 2003; TsaI et al., 2004; ENDRES et al., 2009; GAO et al., 2013). Regarding this, health status of animals, like activity, behaviour, and fur lustre, etc., is the most important indicator to evaluate the acute toxicity of the strain. In this study, no abnormal behaviour such as change in activity or decline in hair lustre occurred in rats fed with the strain E. faecalis HZNU P2 at high dose. All the rats were healthy and survived after 10 days. Furthermore, histopathologic analysis did not show any obvious differences in the appearance of visceral organs between each group. No hepatomegaly, splenomegaly, and any microscopic lesions in renal were observed (Fig. 1). These results indicated that E. faecalis HZNU P2 had no acute oral toxicity effects on general health status of the rats when orally administrated at high dose. This was in accordance with the findings of HUANG and co-workers (2003) and LeE and co-workers (2010).

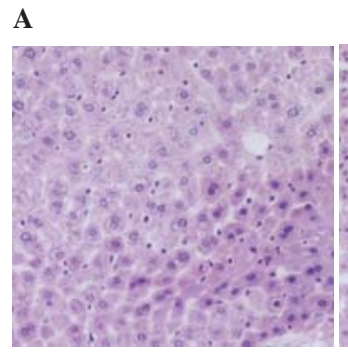

Liver

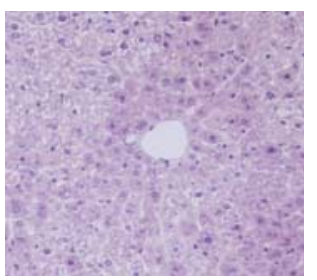

Liver

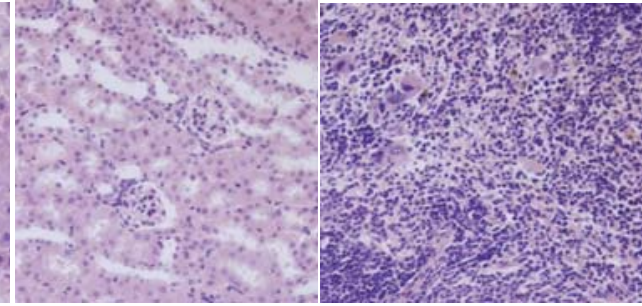

Renal

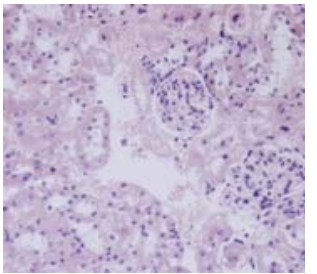

Renal
Spleen

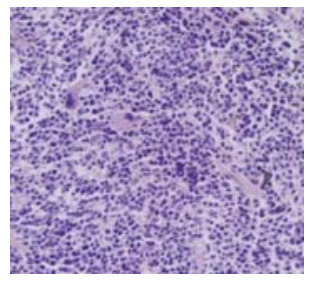

Spleen

Fig. 1. Organs sections from rats treated with high dose of the strain E. faecalis HZNU P2 $\left(2 \times 10^{11} \mathrm{CFU} \mathrm{kg}^{-1}\right)$ after 10 days of treatment

A: Treatment by the strain E. faecalis HZNU P2; B: control group treated by saline solution 


\subsection{Sub-acute toxicity}

A 14 days sub-acute toxicity of this strain was evaluated by administrating the rats with different doses of the strain E. faecalis HZNU P2. During the experimental periods, there was no noticeable change of activity, behaviour, or hair lustre of the rats in any groups. No incidence of diarrhoea, constipation, or other gastrointestinal diseases related to the strain administration was observed. At the end of the experimental period, all rats were alive and healthy. In addition, the change of body weights indicated that the growth of the rats in the treated groups was unaffected by the administration of the strain (Fig. 2). The ratio of organto-body weight is an indicator of organ inflammation. The organ relative weights (organ weight/body weight ratios) in rats are shown in Figure 3. There were no significant differences in relative weights of renal, liver, and spleen among the rats between treatment and control groups $(\mathrm{P}>0.05)$. Infectivity is an important factor in evaluating the safety of probiotic
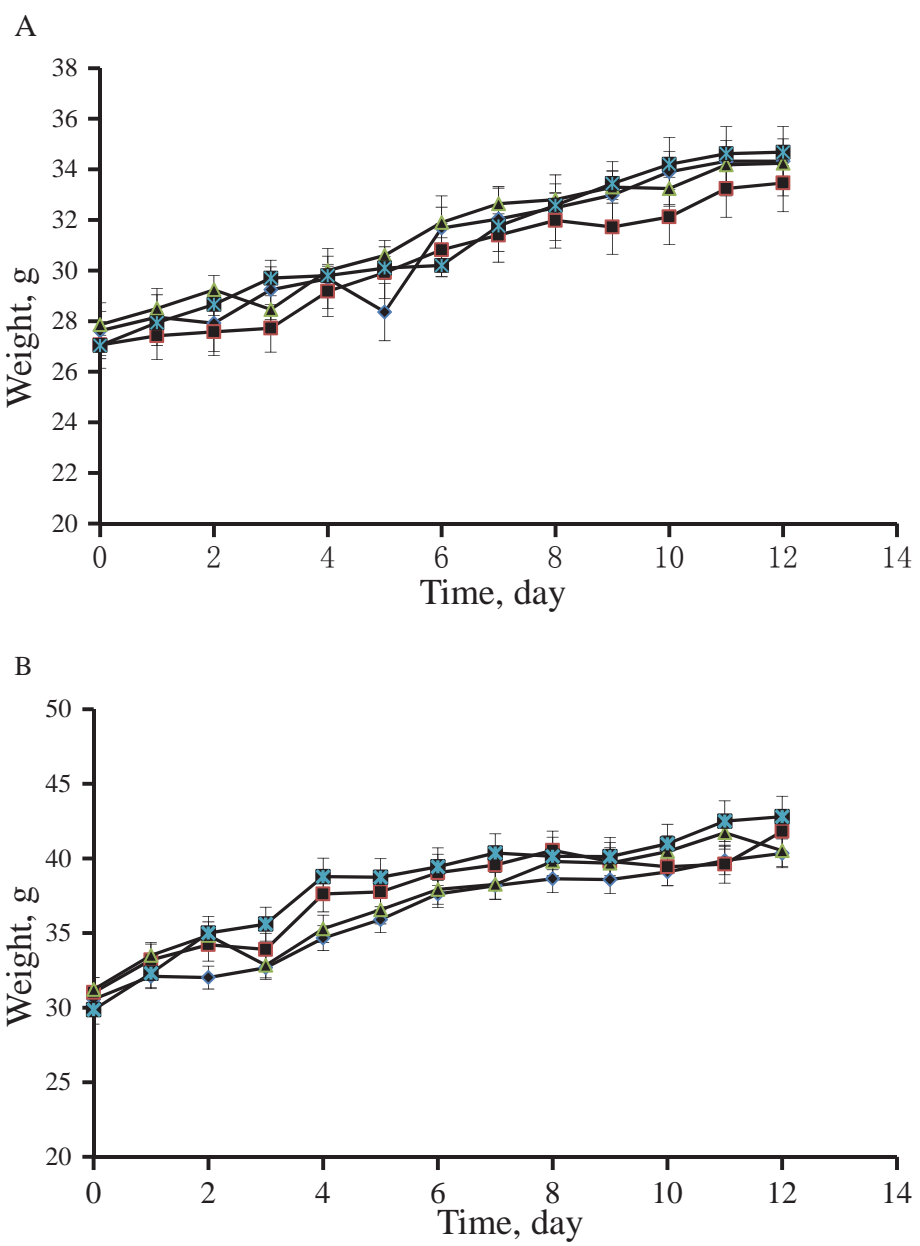

Fig. 2. The body weight change of rats administrated different dosages of the strain E. faecalis HZNU P2 for 14 days, A: Female rats; B: male rats; high dose: $2 \times 10^{11} \mathrm{CFU} \mathrm{kg}^{-1}$; medium dose: $5 \times 10^{10} \mathrm{CFU} \mathrm{kg}^{-1}$; low dose: $1 \times 10^{10} \mathrm{CFU} \mathrm{kg}^{-1}, \diamond$ : Low dose; $\square$ : medium dose; $\triangle$ : high dose; $*$ : control 
bacteria. Splenomegaly and hepatomegaly are indirect indicators of infection. Histopathological examination of renal, liver, and spleen revealed no changes in treatment groups when compared to control group (Fig. 4). No macroscopic pathology findings were observed in any organs. All these results suggest that there are no infections resulting from 14 days treatment with the strain.

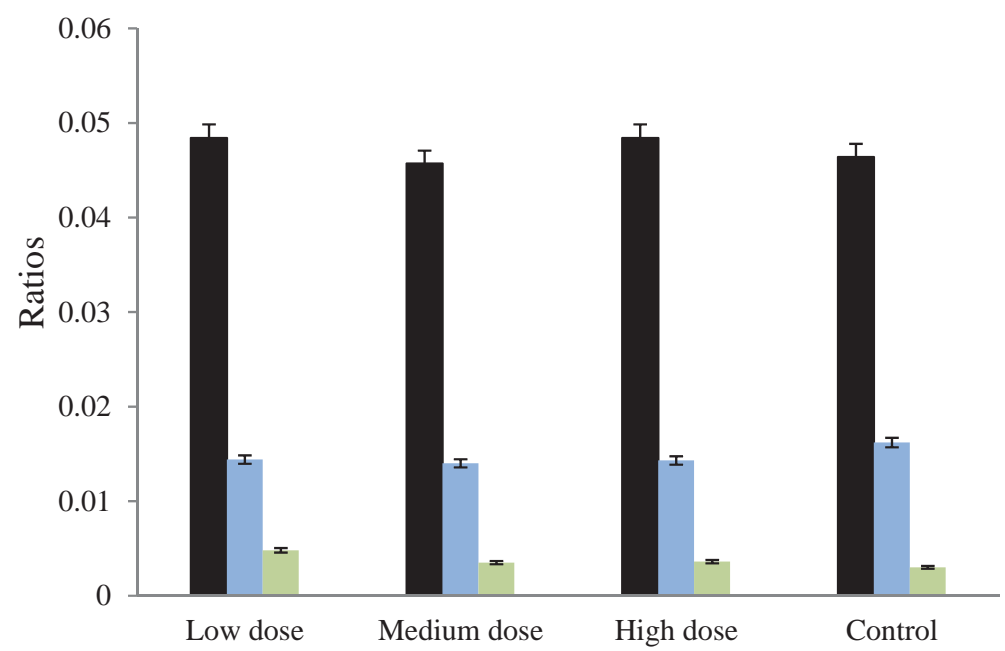

Fig. 3. Organ weight of rats administrated different dosages of the strain E. faecalis HZNU P2 after 14 days of treatment high dose: $2 \times 10^{11} \mathrm{CFU} \mathrm{kg}{ }^{-1}$; medium dose: $5 \times 10^{10} \mathrm{CFU} \mathrm{kg}^{-1}$; low dose: $1 \times 10^{10} \mathrm{CFU} \mathrm{kg}^{-1}$; Control group treated by saline solution. $\square:$ Liver, $\sqsubset:$ renal, $\leftleftarrows:$ : spleen

A

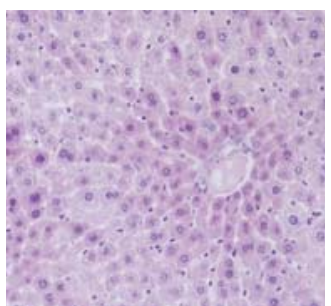

Liver

B

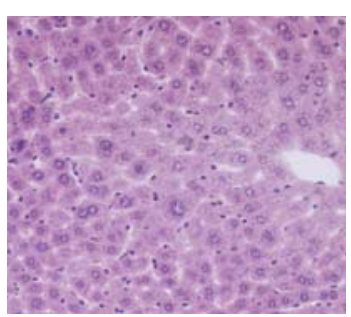

Liver

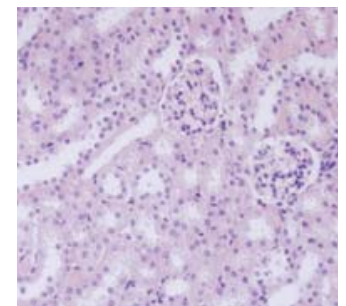

Renal

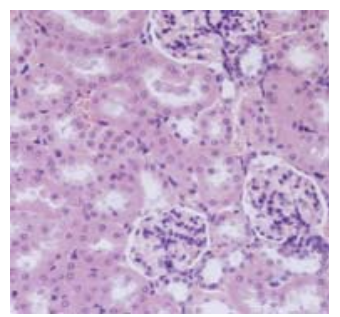

Renal

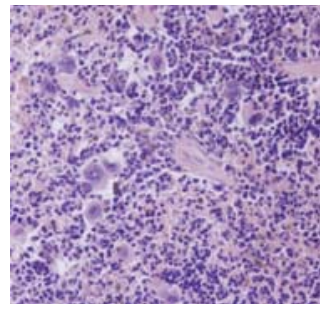

Spleen

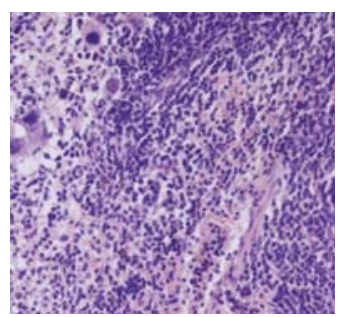

Spleen

Fig. 4. Organs sections from rats treated with high dose of the strain E. faecalis HZNU P2 $\left(2 \times 10^{11} \mathrm{CFU} \mathrm{kg}^{-1}\right)$ after 14 days of treatment

A: Treatment by the strain E. faecalis HZNU P2; B: control group treated by saline solution 


\subsection{Haematological assays}

Haematological assays can be used to determine the effect of the test substance on the blood (Tsai et al., 2004; YAKUBU et al., 2007; LeE et al., 2010). In order to investigate the effect of ingestion of the strain E. faecalis HZNU P2 on blood, some haematological and plasma biochemical parameters were assayed, the findings are shown in Table 1. It was noted that the strain had no adverse effects on concentrations of red blood cells, haemoglobin, white blood cells, lymphocytes, neutrophils, and monocytes. The cholesterol, total protein, triglycerides, and glucose levels in rats treated with the strain were also not significantly different from those of control group. This suggests that treatment of rats with the strain E. faecalis HZNU P2 at high dose had no adverse effects on haematological and plasma biochemical parameters. TSAI and co-workers (2004) also found that administration of E. faecium TM 39 to rats had no effects on haematology and histopathology.

Table 1. Hematologic and plasma biochemical findings in rats treated with the strain E. faecalis HZNU P2 after 14 days of treatment

\begin{tabular}{|c|c|c|c|c|}
\hline Parameter & High dose & Medium dose & Low dose & Control \\
\hline \multicolumn{5}{|l|}{ Male } \\
\hline $\mathrm{RBC}\left(\times 10^{12} 1^{-1}\right)$ & $10.94 \pm 0.03$ & $11.13 \pm 0.07$ & $11.14 \pm 0.06$ & $10.95 \pm 0.08$ \\
\hline $\mathrm{HB}\left(\mathrm{g} \mathrm{l}^{-1}\right)$ & $133.60 \pm 2.3$ & $137.60 \pm 3.3$ & $135.80 \pm 2.7$ & $132.30 \pm 1.9$ \\
\hline WBC $\left(\times 10^{12} 1^{-1}\right)$ & $6.05 \pm .071$ & $5.98 \pm 0.60$ & $6.02 \pm 0.58$ & $6.08 \pm 0.45$ \\
\hline Ly $(\%)$ & $83.17 \pm 2.1$ & $83.58 \pm 1.9$ & $83.62 \pm 1.8$ & $84.02 \pm 2.2$ \\
\hline $\mathrm{Ne}(\%)$ & $7.31 \pm .083$ & $7.12 \pm 0.68$ & $6.89 \pm 0.67$ & $6.86 \pm 0.74$ \\
\hline Mo (\%) & $6.87 \pm 0.52$ & $6.78 \pm 0.49$ & $6.31 \pm 0.48$ & $6.60 \pm 0.42$ \\
\hline $\mathrm{TP}\left(\mathrm{g} \mathrm{l}^{-1}\right)$ & $160.1 \pm 3.4$ & $159.4 \pm 3.2$ & $161.6 \pm 3.5$ & $163.3 \pm 3.6$ \\
\hline $\mathrm{CHO}\left(\mathrm{mmol} \mathrm{l}^{-1}\right)$ & $8.67 \pm 0.23$ & $8.85 \pm 0.34$ & $8.93 \pm 0.37$ & $9.04 \pm 0.42$ \\
\hline GLU $\left(\mathrm{mmol} \mathrm{l}^{-1}\right)$ & $10.5 \pm 0.71$ & $10.3 \pm 0.56$ & $10.8 \pm 0.68$ & $10.1 \pm 0.43$ \\
\hline $\mathrm{TG}\left(\mathrm{mmol} \mathrm{l}^{-1}\right)$ & $4.22 \pm 0.13$ & $4.18 \pm 0.14$ & $4.51 \pm 0.18$ & $4.35 \pm 0.19$ \\
\hline \multicolumn{5}{|l|}{ Female } \\
\hline $\mathrm{RBC}\left(\times 10^{12} 1^{-1}\right)$ & $10.86 \pm 0.04$ & $10.92 \pm 0.06$ & $11.51 \pm 0.08$ & $10.55 \pm 0.12$ \\
\hline $\mathrm{HB}\left(\mathrm{g} \mathrm{l}^{-1}\right)$ & $137.40 \pm 1.9$ & $135.80 \pm 2.1$ & $137.60 \pm 1.5$ & $133.80 \pm 2.5$ \\
\hline $\mathrm{WBC}\left(\times 10^{12} 1^{-1}\right)$ & $6.10 \pm 0.56$ & $6.23 \pm 0.59$ & $6.67 \pm 0.62$ & $5.88 \pm 0.65$ \\
\hline Ly $(\%)$ & $79.81 \pm 2.3$ & $83.73 \pm 3.2$ & $84.72 \pm 3.6$ & $83.81 \pm 2.9$ \\
\hline $\mathrm{Ne}(\%)$ & $6.95 \pm 0.83$ & $7.23 \pm 0.87$ & $7.31 \pm 0.58$ & $7.00 \pm 0.62$ \\
\hline Mo (\%) & $6.63 \pm 0.63$ & $6.79 \pm 0.65$ & $6.45 \pm 0.73$ & $6.77 \pm 0.74$ \\
\hline $\mathrm{TP}\left(\mathrm{g} \mathrm{l}^{-1}\right)$ & $160.7 \pm 3.2$ & $161.4 \pm 3.6$ & $163.5 \pm 3.8$ & $162.3 \pm 2.9$ \\
\hline $\mathrm{CHO}\left(\mathrm{mmol} \mathrm{l}^{-1}\right)$ & $8.74 \pm 0.37$ & $8.63 \pm 0.34$ & $8.85 \pm 0.29$ & $8.98 \pm 0.27$ \\
\hline GLU $\left(\mathrm{mmol} \mathrm{l}^{-1}\right)$ & $9.8 \pm 0.72$ & $10.2 \pm 0.68$ & $10.3 \pm 0.67$ & $10.1 \pm 0.73$ \\
\hline TG $\left(\mathrm{mmol} \mathrm{l}^{-1}\right)$ & $4.13 \pm 0.09$ & $4.20 \pm 0.17$ & $4.53 \pm 0.12$ & $4.35 \pm 0.15$ \\
\hline
\end{tabular}

\section{Conclusions}

Safety assessment of E. faecalis HZNU P2 isolated from peacock faeces was studied in this paper. The results of this study demonstrated that ingestion of E. faecalis HZNU P2 with different dose had no adverse effect on health, growth, haematological and histology parameters in rats. In addition, acute toxicity in rats fed with the strain at high dose was not observed. These results suggest that E. faecalis HZNU P2 is likely to be safe for animal consumption. All the obtained results will build a basis to develop functional products using the strain E. faecalis HZNU P2. 
This study was financially supported by the Xinmiao Talent Program of Zhejiang Province (2012R421003, 2013R421006).

\section{References}

BaI, A.P. \& Ouyang, Q. (2006): Probiotics and inflammatory bowel diseases. Postgrad. Med. J., 82, 376-382. Endres, J.R., Clewell, A., Jade, K.A., Farber, T., Hauswirth, J. \& Schauss, A.G. (2009): Safety assessment of a proprietary preparation of a novel probiotic, Bacillus coagulans, as a food ingredient. Food Chem. Toxicol., 47, 1231-1238.

GaO, Y., Shen, J., Yin, J., Li, C., Fu, C. \& ChO, S. (2013): A subchronic dietary toxicity study of rice hull fiber in rats. Food Chem. Toxicol., 51, 137-142.

Gomes, B.C., Esteves, C.T., Palazzo, I.C.V., Darini, A.L.C., Felis, G.E., Sechi, L.A., Franco, B.D.G.M. \& Martinis, E.C.P.D. (2008): Prevalence and characterization of Enterococcus spp. isolated from Brazilian foods. Food Microbiol., 25, 668-675.

Hamilton-Miller, J.M. (2004): Probiotics and prebiotics in the elderly. Postgrad. Med. J., 80, 447-451.

Huang, Y., Kotula, L. \& Adams, M.C. (2003): The in vivo assessment of safety and gastrointestinal survival of an orally administered novel probiotic, Propionibacterium jensenii 702, in a male Wistar rat model. Food Chem. Toxicol., 41, 1781-1787.

Kirtzalidou, E., Pramateftaki, P, Kotsou, M. \& Kyriacou, A. (2011): Screening for lactobacilli with probiotic properties in the infant gut microbiota. Anaerobe, 17, 440-443.

Laukova, A., Simonova, M., Strompfova, V., Styriak, I., Ouwehand, A.C. \& Varady, M. (2008): Potential of enterococci isolated from horses. Anaerobe, 14, 234-236.

LeE, K.C., Liu, C.F., Lin, T.H. \& PAN, T.M. (2010): Safety and risk assessment of the genetically modified lactococci on rats intestinal bacterial flora. Int. J. Food Microbiol., 142, 164-169.

Lin, W.H., Yu, B., JANG, S.H. \& Tsen, H.Y. (2007): Different probiotic properties for Lactobacillus fermentum strains isolated from swine and poultry. Anaerobe, 13, 107-113.

Linaje, R., Coloma, M.D., Perez-Martinez, G. \& Zuniga, M. (2004): Characterization of faecal enterococci from rabbits for the selection of probiotics strains. J. Appl. Microbiol., 96, 761-771.

Mach, T. (2006): Clinical usefulness of probiotics in inflammatory bowel diseases. J. Physiol. Pharmacol., 57, 2333.

Martin, R., Soberon, N., Vaneechoutte, M., Florez, A.B., Vazquez, F. \& Suarez, J.E. (2008): Characterization of indigenous vaginal lactobacilli from healthy women as probiotic candidates. Int. Microbiol., 11, 261-266.

McFarland, L.V. \& Dublin, S. (2008): Meta-analysis of probiotics for the treatment of irritable bowel syndrome. World J. Gastroentero., 14, 2650-2661.

Medina, R., Katz, M., Gonzalez, S. \& Oliver, G. (2001): Characterization of the lactic acid bacteria in ewe's milk and cheese from northwest Argentina. J. Food Protect., 64, 559-563.

NAKAI, S.A. \& SieBerT, K.J. (2003): Validation of bacterial growth inhibition models based on molecular properties of organic acids. Int. J. Food Microbiol., 86, 249-255.

OuweHAND, A.C. (2007): Antiallergic effects of probiotics. J. Nutr., 137, 794S-797S.

Safdar, A., Bryan, C.S., Stinson, S. \& Saunders, D.E. (2002): Prosthetic valve endocarditis due to vancomycinresistant Enterococcus faecium: treatment with chloramphenicol plus minocycline. Clin. Infect. Dis., 34, E61E63.

Sнан, N.P. (2007): Functional cultures and health benefits. Int. Dairy J., 17, 1262-1277.

Shi, L.E., Li, Z.H., Li, D.T., Xu, M., Chen, H.Y., Zhang, Z.L. \& TANG, Z.X. (2013): Encapsulation of probiotic Lactobacillus bulgaricus in alginate-milk microspheres and evaluation of survival in simulated gastrointestinal conditions. J. Food Eng., 117, 99-104.

Soler, J.J., Martin-Vivaldi, M., Rui-Rodriguez, M., Valdivia, E., Martin-Platero, A. M., Martinez-Bueno, M., Peralta-Sanchez, J.M. \& Mendez, M. (2008): Symbiotic association between hoopoes and antibioticproducing bacteria that live in their uropygial gland. Funct. Ecol., 22, 864-871.

Tsai, C.C., Liu, T.H., Chen, M.H., Tsai, C.C. \& Tsen, H.Y. (2004): Toxicity evaluation for an Enterococcus faecium strain TM39 in vitro and in vivo. Food Chem. Toxicol., 42, 1601-1609.

Yakabe, T., Moore, E.L., Yokota, S., Sui, H., Nobuta, Y., Fukao, M., Palmer, H. \& Yajima, N. (2009): Safety assessment of Lactobacillus brevis KB290 as a probiotic strain. Food Chem. Toxicol., 47, 2450-2453. 
Yakabe, T., Takashima, H., Kuwagata, M., Fukao, M., Kikuchi, S. \& Yajima, N. (2011): Teratogenicity and maternal effects of Lactobacillus brevis KB290 in rats and rabbits. Food Chem. Toxicol., 49, 722-726.

Yakubu, M.T., Akanji, M.A. \& Oladis, A.T. (2007): Haematological evaluation in male albino rats following chronic administration of aqueous extract of Fadogia agrestis stem. Pharmacogn. Mag., 3, 34-38.

Zheng, W., Zhang, Y., Lu, H.M., Li, D.T., Zhang, Z.L., TAng, Z.X. \& Shi, L.E. (2015): Antimicrobial activity and safety evaluation of E. faecium KQ 2.6 isolated from peacock feces. BMC Biotechnol., 15, 30

Zhou, J.S., Shu, Q., Rutherfurd, K.J., Prasad, J., Birles, M.J., Gopal, P.K. \& Gill, H.S. (2000): Safety assessment of potential probiotic lactic acid bacterial strains Lactobacillus rhamnosus HN001, Lb. acidophilus HN017, and Bifidobacerium lactis HN019 in BALB/c mice. Int. J. Food Microbiol., 56, 87-96. 